\title{
Docentes, discentes y trabajos académicos: Propiedad Intelectual en conflicto $^{1}$
}

Teachers, students and academic works:

Intellectual Property in conflict

\section{Francisca Ramón Fernández}

Profesora titular de Derecho civil

Escuela Técnica Superior de Ingeniería Informática (ETSII)

Universitat Politècnica de València

Email: frarafer@urb.upv.es

\author{
Vicent Giménez Chornet \\ Profesor titular de Documentación \\ Escuela Técnica Superior de Ingeniería Informática (ETSII) \\ Universitat Politècnica de València \\ Email: vigicho@har.upv.es
}

\section{Juan Vicente Oltra Gutiérrez}

Profesor titular de Organización de Empresas

Escuela Técnica Superior de Ingeniería Informática (ETSII)

Universitat Politècnica de València

Email: jvoltra@omp.upv.es

\footnotetext{
${ }^{1}$ Trabajo realizado en el marco del Proyecto I+D+i «Retos investigación» del Programa estatal de I+D+i orientado a los Retos de la Sociedad del Ministerio de Ciencia, Innovación y Universidades: RTI2018-097354-B-100 (2019-2022), Proyecto de I+D+i Retos MICINN "Derechos y garantías frente a las decisiones automatizadas en entornos de inteligencia artificial, IoT, big data y robótica" (PID2019-108710RB-I00, 2020-2022). Investigador principal: Dr. D. Lorenzo Cotino Hueso. Catedrático de Derecho Constitucional. Universitat de València, y en el Proyecto de Innovación y Mejora Educativa (PIMEs) concedido por el Vicerrectorado de Estudios y Convergencia Europea de la Universitat Politècnica de València (2018-2020).
} 
Resumen: La participación del profesorado en la dirección de trabajos en el ámbito universitario plantea diversas cuestiones en relación con la protección de los derechos de autor. La orientación, líneas a seguir, así como la dedicación e implicación en la redacción del trabajo del alumnado no se contemplan como objeto protegido por la propiedad intelectual, ni en la legislación aplicable, ni en la normativa interna de la Universitat Politècnica de València. Proponemos en este trabajo una reflexión sobre la participación del profesorado, un replanteamiento de la definición de dirección, así como la redacción de un contrato entre alumnado y profesorado para delimitar la participación y protección de la obra.

Palabras clave: Trabajos académicos; dirección y tutorización del profesorado; tesis de grado; derechos de autor; investigación; autoría; Universidad.

\begin{abstract}
The participation of the teaching staff in the direction of work in the university field raises several questions in relation to the protection of copyright. The orientation, lines to follow, as well as the dedication and involvement in the writing of the students' work are not considered as an object protected by intellectual property, neither in the applicable legislation nor in the internal regulations of Polytechnic University of Valencia. We propose in this work a reflection on the participation of teachers, a rethinking of the definition of direction, as well as the drafting of a contract between students and teachers to delimit the participation and protection of the work.
\end{abstract}

Keywords: Academic work; direction and tutoring of teachers; undergraduate dissertations; copyright; research; authorship; University. 


\section{Algunas consideraciones}

El Espacio Europeo de Educación Superior ha propiciado que tanto alumnado como profesorado asuman distintos roles (Da Silva, 2007: 102; Canós et al., 2008: 388). Sin embargo, el incremento de tareas o trabajos evaluativos en las asignaturas no ha significado una mejora sustancial en el aprendizaje del alumnado que, en la mayoría de las ocasiones, se enfrenta al trabajo final de su titulación con muchas carencias, y todo ello a pesar de haberse incrementado las herramientas digitales para facilitar el autoaprendizaje (Ramón, 2014: 85; Lull et al., 2017: 390). Ello no ocurre sólo en el Grado, sino también en Máster y Doctorado, en el que la dirección del profesorado se torna en una actividad que le lleva a implicarse de forma muy directa en la elaboración de los manuscritos (López, 2008: 25; 2010: 30). Es preciso indicar que las condiciones en las que se realiza el trabajo académico divergen según se encuentre el alumnado en Grado, Máster o Doctorado, y que la autonomía del alumnado debe aumentar de forma progresiva, pero ello no siempre es así (Buendía et al., 2017: 215; Allen et al., 2018: 297). Resulta de interés determinar qué contenidos deben ser los esenciales en el aprendizaje del alumnado, así como las herramientas e instrumentos que debe conocer para desarrollar un trabajo académico (Camilloni, 2019: 7).

Esta participación del profesorado en la dirección de trabajos en el ámbito universitario plantea diversas cuestiones en relación con la protección de los derechos de autor (Canós et al., 2007: 8). Hay que tener en cuenta que el profesorado interviene en distintos aspectos: orientación, líneas a seguir, redacción, ayuda bibliográfica y diseño de la estructura. Los trabajos académicos al final son depositados en repositorios digitales en abierto, y se requiere especial atención en algunos temas, especialmente en cuanto a la propiedad intelectual y la calidad de los mismos (Schopfel, et al., 2018).

Estos trabajos son el Capital Intelectual de la organización que los ha producido, en este caso la Universidad. La gestión de esa propiedad intelectual es relevante, y debemos considerar el gran valor que reúne como activo intangible en la transferencia tecnológica de la universidad, que posee derechos legales o contractuales asociados (Loyarte et al., 2018). 
Nos podemos plantear si existe un "agujero en la norma", ya que la actividad del profesorado en relación con la participación en la dirección de los trabajos no se contempla como objeto protegido por la propiedad intelectual; no se indica dicha actividad en la legislación aplicable, ni se suele contemplar en las normativas internas (Universidades, Estructuras Responsables del Título-ERT's-). Sin embargo diversos informes destacan el capital intelectual de las universidades europeas nacido de las actividades de investigación, siendo un reto medir con indicadores esas actividades de investigación (Secundo et al., 2010), algunas de ellas plasmadas en Trabajos Final de Carrera o en Tesis Doctorales.

Si bien es verdad que en algunas ocasiones la doctrina se ha ocupado de dar difusión a recomendaciones, fórmulas para redactar correctamente, o pautas diversas para realizar los trabajos (Mikelarena, 2007: 239; Toller, 2010: 489; Lahoz, 2012: 20; Ubertone, 2017: 245), es una quimera considerar que el alumno puede hacer el trabajo sin ningún tipo de asesoramiento por parte del personal docente e investigador (Canós et al., 2009: 345).

Nos proponemos en el presente trabajo reflexionar sobre la participación del profesorado en la dirección de trabajos académicos, en cualquier nivel, tanto en grado, postgrado como en doctorado. Incidir en un replanteamiento de la definición de "dirección", la posibilidad de canalizar la dirección a través de la redacción de un contrato alumnadoprofesorado, para determinar los límites, así como la participación y protección de la obra.

\section{La dirección del profesorado (Trabajo Final de Grado, Trabajo Final de Máster, Tesis Doctoral): Casuística: mínima implicación versus máxima implicación del profesorado}

La implicación del profesorado universitario en los trabajos de investigación que dirige, ya sean Trabajo Final de Grado, Tesis Final de Máster o Tesis Doctorales, puede ser de diversa índole, lo que conlleva a una difícil delimitación en el resultado final de la autoría de la obra, y por tanto en el grado de derechos de autor compartidos. Por parte del alumno, éste debe haber conseguido una alfabetización en información que le permita desarrollar una línea básica de investigación usando los recursos que se disponen en las 
bibliotecas y otros centros de investigación con abundantes fuentes de información (Licea de Arenas, et. al., 2004).

Puede haber diversas tipologías de casos en el inicio de un trabajo de investigación, que el alumno finalmente redactará, con corrección o no del profesor. El caso más alejado de la involucración del profesor en el resultado final del trabajo de investigación es cuando el propio alumno sugiere la idea a investigar, utiliza la metodología correcta de análisis, consulta la bibliografía y fuentes de información adecuadas para contextualizar, justificar y argumentar su investigación, expone los resultados de forma pertinente a los objetivos de la investigación, la redacción no necesita de correcciones, y llega a unas conclusiones adecuadas a su análisis. En las últimas décadas ha habido una creciente preocupación por fomentar la calidad de los trabajos final de grado o máster, y esta preocupación generalizada se ha dirigido principalmente hacia una mayor responsabilidad e implicación de los profesores, cada vez más atentos al resultado final del trabajo y a una mayor participación en su evaluación (Pathirage, et. al., 2007).

Con la experiencia de muchos trabajos dirigidos el caso del profesor alejado de la investigación del alumno es casi nulo, aunque ha habido algún alumno aventajado a nivel de Trabajo Final de Grado que, tras el acuerdo entre el profesor y el alumno sobre un tema a trabajar, y una estructura del análisis, el alumno no ha necesitado más orientación y ha terminado el Trabajo Final de Grado con éxito. Pero esto no nos ha ocurrido a nivel de Trabajo Final de Máster y Tesis Doctoral.

Tal y como indica la doctrina nos podemos encontrar con diferentes tipologías de dirección, estilos de tutorización e incluso modalidades de evaluación (Padilla et al., 2008: 843; Pérez, 2008: 157; Pacenza et al., 2017: 40; Romero, 2017: 735; Vera, 2017: 277; Díaz-Vázquez et al., 2018: 159).

La casuística más frecuente cuando un alumno quiere iniciar un trabajo de investigación es que el profesor se vea involucrado en los siguientes puntos:

a)

El profesor sugiere y acota el tema específico de la investigación, tal vez a partir de una idea extremadamente amplia del conocimiento o materia, resaltando lo que 
es tema de investigación porque no se conocen los resultados, y descartando lo que no es tema de investigación porque ya es conocido (y esto es posible por la experiencia del profesor).

b)

Una vez escogido el tema es el profesor quien suele delimitar los objetivos, vinculados a la importancia del trabajo que hará el alumno (ya sea Trabajo Final de Grado, Trabajo Final de Máster o Tesis Doctoral), para que éste pueda realizar con éxito su investigación en el tiempo y créditos docentes requeridos.

c)

El profesor le sugiere la metodología a seguir, de la cual el alumno suele ser bastante inexperto, tal vez por la falta de práctica en los cursos de grado e incluso de posgrado, dado que prácticamente nunca han redactado un artículo científico. La dirección del profesor llega incluso a delimitar la metodología más pertinente para cada caso en particular (Confrontar: Narváez, 2019: 82).

d) El profesor es el que suele disponer de una visión global de la bibliografía y fuentes de información pertinentes sobre el tema escogido, y orienta al alumno en qué repositorios bibliográficos pertinentes debe de completar la búsqueda de información que garantizará la contextualización, planteamientos y sustentación del resultado final. De hecho, las nuevas tecnologías de la información y comunicación resultan fundamentales para la elaboración de cualquier trabajo académico (Cicero, 2018:95). También interesa destacar que el profesor orientará al alumnado sobre cuáles son los repositorios adecuados para consultar, evitando que pueda obtener información falsa o poco fiable, que pueda perjudicar la investigación (Marín, 2017:99). Asimismo, también le orientará sobre los materiales libres y los materiales sujetos a propiedad intelectual, y las limitaciones a su utilización (Urías, 1999: 442).

e)

Otro aspecto a resaltar es la indicación por parte del profesor de la utilización de la jurisprudencia para complementar el trabajo, en los casos en que proceda, así como desarrollar el espíritu crítico, indicándole al alumno las bases de datos y repositorios que puede utilizar para su consulta, teniendo en cuenta las características de cada uno de ellos, ya sean de libre acceso, o bien con licencia, y en todo caso la cita correspondiente de la fuente de obtención de la misma (Verdera, 2014: 9). 

resultados suele estar orientada por el profesor, para que estos resultados sean lo más explícitos posibles, descartando conocimiento redundante.

El profesor suele orientar al alumno para que redacte unas conclusiones acordes a los objetivos de investigación. Es frecuente que el alumno redacte frases de conclusiones que van más allá del objeto de investigación planteado.

h) El profesor supervisa, corrige, e incluso llega a incluir algún párrafo, en la redacción final del trabajo de investigación. Esto es muy frecuente porque el alumno no suele tener experiencia en el uso del vocabulario científico.

i)

Además, también el profesor orienta al alumno sobre la exposición oral del trabajo, incluso realizando ensayos previos, para ver su oralidad y dominio de la exposición, incluso dándole pautas para realizar una presentación óptima (Navarro y Melero, 2018: 21).

A diferencia de lo anterior, la implicación del profesorado puede ser mínima porque el alumno sugiere el tema que va a investigar; utiliza la metodología correcta de análisis, es autónomo en la consulta de bibliografía, conoce las fuentes y su localización; es capaz de contextualizar, justificar y argumentar su investigación de forma independiente; expone de forma pertinente los resultados, y no necesita correcciones la redacción del texto, y llega a unas conclusiones adecuadas a su análisis.

Estos casos son prácticamente nulos en la actualidad.

No obstante, en algunos casos, tanto en la máxima implicación como en la mínima, el profesor puede encontrarse ante situaciones en las que propone y delimita el tema de investigación /proyecto; en la que supervisa, corrige e incluso llega a incluir párrafos, reformula lo escrito, y pone su aporte en el trabajo de investigación. Esto es muy frecuente porque el alumno no suele tener experiencia en el uso del vocabulario científico. Incluso se llega al punto de que el profesor hace de corrector ortográfico, ya que en muchas ocasiones los trabajos contienen errores, y ello conlleva que la corrección vaya 
más allá de la puramente académica, y se involucre el profesorado en la redacción, ortografía y sintaxis del texto (Melguizo, 2016: 521).

Con todas estas variables la cuestión es ¿cómo se puede medir o evaluar el grado de intervención del profesor en la redacción final de una investigación? ¿Hay alguna solución que refleje objetivamente la involucración del profesor en el trabajo de investigación del alumno? ¿El profesor no debe transferir su conocimiento científico al alumno, y dejar que este realice solo los puntos indicados más arriba? ¿No "hacemos trampa" si participamos demasiado del "producto final"? Ahí surge la difícil delimitación en el resultado final de la autoría de la obra, y la complejidad de determinar el grado de derechos de autor compartidos.

\section{Marco jurídico actual y jurisprudencia}

Respecto a la regulación en el ordenamiento jurídico español relacionado con los derechos de propiedad intelectual, podemos indicar la siguiente normativa que consideramos oportuna, sin ánimo exhaustivo (más ampliamente, Gómez, 2018: 209):

1. El Acuerdo de la Ronda Uruguay: Aspectos de los derechos de propiedad intelectual relacionados con el comercio (ADPIC), Anexo 1C del Acuerdo de Marrakech por el que se establece la Organización Mundial del Comercio, firmado en Marrakech, Marruecos, el 15 de abril de 1994, cuyo artículo 9 indica que la protección del derecho de autor abarca las expresiones, pero no las ideas.

2. Convenio de Berna para la Protección de las Obras Literarias y Artísticas, de 9 de septiembre de 1886 , en su artículo 2 precisa que los términos obras literarias y artísticas incluyen todas las producciones en el campo literario, científico y artístico, con independencia de su modo o forma de expresión.

3. Ley 2/2011, de 4 de marzo, de Economía Sostenible ${ }^{2}$, indica en el artículo 55, que en la transmisión a terceros de derechos sobre los resultados de la actividad investigadora

\footnotetext{
${ }^{2}$ Boletín Oficial del Estado núm. 55, de 5 de marzo de 2011.
} 
(transmisiones y contratos relativos a la propiedad intelectual) se aplicará el derecho privado.

4. Real Decreto Legislativo 1/1996, de 12 de abril, por el que se aprueba el texto refundido de la Ley de Propiedad Intelectual, regularizando, aclarando y armonizando las disposiciones legales vigentes sobre la materia ${ }^{3}$, en la que se establece que la propiedad intelectual corresponde al autor por el solo hecho de crearla (artículo 1), y se integra por derechos personales y patrimoniales (artículo2). Se considera autor la persona natural que crea la obra (artículo 5), y se contempla la obra en colaboración (artículo 7).

5. Real Decreto 1791/2010, de 30 de diciembre, por el que se aprueba el Estatuto del Estudiante Universitario ${ }^{4}$, en el que se establece como derechos del alumnado, en el artículo $8 \mathrm{~h}$ : «contar con el reconocimiento y protección de la propiedad intelectual del trabajo fin de grado y de los trabajos previos de investigación en los términos que se establecen en la legislación vigente sobre la materia», y el artículo 9 h, «contar con el reconocimiento y protección de la propiedad intelectual del trabajo fin de máster y de los trabajos previos de investigación en los términos que establecen en la legislación vigente sobre la materia».

Incidir en que el criterio que se aplica es que haya originalidad, para delimitar los trabajos que son protegidos por la propiedad intelectual de los que no. Este concepto que aparece en el artículo 10 del Real Decreto Legislativo 1/1996, al que hemos hecho referencia es, como señala Llopis (2019:4) «un concepto jurídico cuya determinación ha de realizarse estando a las circunstancias que presente el trabajo de investigación concreto. Para ello debe tenerse en cuenta que la propiedad intelectual no protege las ideas, sino la forma de expresarlas, por lo que el carácter original no se desprende de la aportación que se realiza a la ciencia jurídica con la obra. Lo indicado no deja de ser paradójico, pues lo más "original" que puede contener un trabajo de investigación es su contenido: la idea con la que el autor contribuye al progreso de la ciencia». El derecho de autor es un derecho legal que garantiza que la propiedad intelectual de la obra realizada por un individuo o una

${ }^{3}$ Boletín Oficial del Estado núm. 97, de 22 de abril de 1996.

${ }^{4}$ Boletín Oficial del Estado núm. 318, de 31 de diciembre de 2010. 
organización esté protegida contra la duplicación o adaptación ilegal de su trabajo, y algunos estudios han alertado a los profesores de las universidades de EE. UU. a ejercer un mayor control de los derechos de autor del trabajo realizado para sus instituciones (conferencias, programas de estudio, materiales de enseñanza, etc.) (Chauhan and Willet, 2019).

En el caso de la Universitat Politècnica de València, la normativa aplicable a dicha Universidad representada por la Normativa Marco de Trabajo Fin de Grado y Fin de Máster aprobada por el Consejo de Gobierno en su sesión de 7 de marzo de 2013 y modificado posteriormente el 29 de enero de 2015, 28 de mayo de 2015, 21 de diciembre de 2017 y 13 de marzo de 2018, indica una serie de precisiones respecto a la realización de los trabajos (arts. 3 y 4), así como los deberes de los tutores y cotutores (art. 9). ${ }^{5}$

De igual modo, en el documento "Guía de buenas prácticas para la dirección de tesis doctorales en la Universitat Politècnica de València”, por la Comisión de Doctorado de 25 de septiembre de 2012, se indica al respecto de la protección y publicación de resultados una serie de directrices respecto a la protección de los mismos, así como la importancia de la figura del director como orientador de la trayectoria del alumnado. ${ }^{6}$

\footnotetext{
${ }^{5}$ «Art. 3, Los TFG y TFM consistirán en la realización de un trabajo o proyecto original en el que queden de manifiesto conocimientos, habilidades y competencias adquiridas por el estudiante a lo largo de sus estudios y, expresamente las competencias asociadas a la materia TFG o TFM, tal y como se indique en la memoria de verificación.
}

Art. 4. 1, Cuando parte o la totalidad de los ECTS asignados a la materia se organicen mediante trabajo autónomo y tutelado, para la realización de su TFG o TFM, los estudiantes contarán con la dirección de un tutor académico que supervisará el trabajo académico y les dará apoyo en la gestión administrativa.

Art. 9, Serán deberes de los tutores y cotutores

* Tutelar al alumno, efectuando un seguimiento periódico de su labor.

* Facilitar al alumno las orientaciones e indicaciones adecuadas para el desarrollo de su trabajo.

* Velar por la calidad y la originalidad el TFG o TFM.

* Reconocer adecuadamente las aportaciones del alumno en los resultados que se deriven de su TFG o TFM».

${ }^{6}$ «El director velará para que el proceso formativo como investigador del doctorando cubra todas las etapas (documentación y formación adecuada en la temática de la tesis, planificación racional de la investigación y 
En este orden, mencionar que en el seno de la Universitat Politècnica de València respecto a los repositorios institucionales, se ha elaborado el acuerdo marco de cesión de derechos de autor de obras digitales en RiuNet, el contrato de depósito de tesis doctorales y la autorización para la difusión de trabajos académicos (Ramón, 2020).

En ningún caso se contempla la propiedad intelectual del director académico del trabajo, siendo estos documentos únicamente el alumnado quien debe de firmarlos a efectos de los derechos de propiedad intelectual.

La jurisprudencia se ha pronunciado sobre el objeto de la propiedad intelectual en diversas ocasiones. Podemos indicar la Sentencia del Tribunal Supremo núm. 647/2012, de 8 de noviembre, en la que precisó que la obra científica es susceptible de protección como objeto el derecho de autor, pero no por su contenido, sino por tener una forma de exposición original. Es por tanto «la forma literaria o artística de su expresión» lo que es objeto de protección. ${ }^{7}$

Como se desprende de la citada sentencia, el Alto Tribunal diferencia el contenido de la forma expositiva de la obra, por lo que la creación científica se protege en tanto en cuanto se muestre de una determinada forma.

protección, comunicación y publicación de los resultados), potenciando la de protección y publicación de resultados.

El director valorará, a lo largo del proyecto de tesis, los resultados obtenidos en cada uno de los objetivos parciales por su posible protección con patente, su comunicación a congresos o su publicación en revistas científicas y de divulgación.

De esta forma, la realización de la tesis llevará asociada de forma simultánea la protección de los resultados, en caso de que sea aconsejable, y una determinada producción científica en forma de comunicaciones y publicaciones científicas.

El director potenciará la participación del doctorando en congresos internacionales en el tema de la tesis, con la presentación de los resultados, para desarrollar sus capacidades de comunicación en entornos de expertos, a ser posible en el idioma más habitual utilizado en la comunicación científica de ese ámbito».

7 Así, precisó que: «Las creaciones científicas no son objeto de propiedad intelectual, por razón de su contenido -ideas, procedimientos, sistemas, métodos operativos, conceptos, principios, descubrimientos...ni de la formación o experiencia de quienes las realizan, impulsan o de los esfuerzos de quienes las financian, sino sólo por la forma literaria o artística de su expresión». 
Respecto al alcance de la protección del derecho de autor y la originalidad de un Proyecto Final de Carrera y un Proyecto Final de Máster en controversia con un libro de los directores de los primeros, la Audiencia Provincial de Valladolid, en sentencia núm. 215/2016, de 1 de julio se indicó que lo verdaderamente relevante para la delimitación de la esfera de protección no es el logro o novedad científica que la obra pueda transmitir, sino la singularidad u originalidad. Y siguió precisando el Tribunal que: «la concreta forma, exteriorización o manifestación literaria utilizada por su autor para la transmisión exclusivamente se extiende sobre la originalidad de las creaciones, y no sobre todos los aspectos o contenidos de las mismas». ${ }^{8}$

Respecto a la originalidad la casuística que llega a los tribunales es muy diversa. El deslinde de lo que es plagio de la obra original tiene unos límites que son, en ocasiones, difíciles de delimitar. Así, podemos citar, a título de ejemplo el caso resuelto por la Audiencia Provincial de Valladolid, en sentencia núm. 384/2015, de 18 de diciembre, en el que la demandante indica que su Proyecto Final de Carrera y Proyecto Final de Máster había sido objeto de plagio por sus directores. El Tribunal se pronuncia acerca de lo que se considera «integración del acervo cultural generalizado». ${ }^{9}$

${ }^{8}$ Entendió el tribunal que: «Los dos trabajos elaborados por la actora carecen de la originalidad necesaria.

Nos hallamos ante dos trabajos universitarios en los que la autora reproduce literalmente, o adapta en el mejor de los casos, los textos que le sirven de fuente, sin ser rigurosa con el derecho a la cita.

En consecuencia, las obras de la actora no son originales, pues su redacción carece del mínimo de singularidad relevante, al presentar, en su práctica totalidad, una redacción semejante, próxima, o fácilmente identificable o reconocible con las fuentes que constituyen referencia en la materia. No dudamos que los trabajos puedan ser útiles para un especialista en la materia, o que el enfoque novedoso o esfuerzo invertido deba ser objeto de consideración, o incluso que merezcan una alta calificación respecto al aprovechamiento académico de la alumna, pero desde una perspectiva literaria o artística su aportación es muy escasa o nula, por lo que no merece una especial protección en el ámbito del derecho de autor.

En definitiva, nos encontramos ante la paradoja de que aquella persona que reclama para su obra la protección dispensada por el derecho de autor, ha utilizado de forma sistemática y reiterada expresiones, frases o gráficos semejantes, próximas o parecidas a las que recogen sus fuentes, privando con ello a su obra de la originalidad necesaria que la haga merecedora de la protección que se impetra».

${ }^{9}$ De esta forma, considera que: «la demandante para hacer sus trabajos se valió de los mismos y de todo el material (apuntes del Máster, referencias bibliográficas, transparencias...) facilitado por las mismas personas, esto es sus profesores del Máster y tutor, aquí curiosamente demandados.

Careciendo de originalidad los trabajos de la demandante, tanto en cuanto al contenido -ausencia de innovación en el estado de la ciencia- como en cuanto a la forma de expresión literaria- transcripción literal 
Otro supuesto respecto a la originalidad y protección de un Trabajo Final de Máster se plantea en la sentencia de la Audiencia Provincial de Murcia núm. 444/2016, de 14 de julio, en la que el Tribunal distingue entre la obra original, que refleja la personalidad del autor, fruto de su creación y no del copiado de obra ajena, y originalidad como novedad objetiva, en cuanto creación de algo nuevo, no preexistente, debiendo tener una relevancia mínima o altura creativa. En el mismo sentido se pronuncian las Sentencias Tribunal Supremo 20 de febrero de 1992; 26 de octubre de 1992 y 24 de junio de 2004. Este problema se plantea también en relación con el plagio del que se ha ocupado la doctrina en varias ocasiones (Toller, 2011: 88; Comás et al., 2011; 361; Ramón, 2013: 245), y el derecho de cita que contempla el art. 32 del TRLPI (Prósper y Ramón, 2019: 130).

Se consideró, pues, en este caso que la protección de la propiedad intelectual de las creaciones científicas, entre las que se encuentran los Trabajos Final de Grado y los Trabajos Final de Máster, exclusivamente se extiende sobre la originalidad de las creaciones y no sobre todos los aspectos o contenidos de las mismas, no protegiendo el derecho de autor las ideas, sino la forma en que las mismas aparecen recogidas en la obra.

En la sentencia de la Audiencia Provincial de Valencia núm. 13, de 21 de enero de 2009, se plantea el caso de un Catedrático que dirige una tesis doctoral y luego utiliza en una pequeña publicación material, conclusiones y estructura de la obra de su doctoranda, y no la cita.

La Audiencia resolvió, apelando a las Sentencias del Tribunal Supremo 28 de enero de 1995; 17 de octubre de 1997 y 23 de marzo de 1999, respecto a lo que se entiende por plagio. $^{10}$

de fuentes bibliográficas en su redacción en inglés, trasunto literal de autores anglófonos, como en la utilización de figuras ilustrativas, e insistiendo en que no desmerecemos la labor recopilatoria de la demandante pero meramente dirigida a demostrar un aprovechamiento académico, sobre la base además de las propias enseñanzas y referencias facilitadas por sus profesores y tutor aquí demandados, y sin que sea objeto de esta litis verificar la originalidad del manual de los demandados (no en cuanto a la actora obviamente, sino respecto de los mismos autores extranjeros, fuente del trabajo, si bien se reconoce por aquellos que es una obra docente sin mayores pretensiones y además mucho más amplia), en modo alguno cabe hablar de plagio».

${ }^{10}$ Considera el Tribunal que «Por plagio hay que entender, en su acepción más simplista, todo aquello que supone copiar obras ajenas en lo sustancial, presentándose más bien como una actividad material 


\section{Propuesta de documento contrato}

\section{Para prevenir los posibles conflictos relacionados con la participación del profesorado} universitario en las investigaciones escritas por los alumnos, en los que el profesor indica al alumno verbalmente diferentes cuestiones, como qué va a investigar, qué objetivos deberá abordar, cómo va a realizar la investigación, que fuentes consultar para conocer el estado del arte, e incluso la corrección del trabajo escrito, proponemos que se redacte un documento entre ambas partes. Se trata de un documento que pretenda ser útil en las

mecanizada y muy poco intelectual y menos creativa, carente de toda originalidad y de concurrencia de genio o talento humano, aunque aporte cierta manifestación de ingenio.

Las situaciones que representan plagio hay que entenderlas como las de identidad, así como las encubiertas, pero que descubren, al despojarlas de los ardides y ropajes que las disfrazan, su total similitud con la obra original, produciendo un estado de apropiación y aprovechamiento de la labor creadora y esfuerzo ideario o intelectivo ajeno. No procede confusión en todo aquello que es común e integra el acervo cultural generalizado o con los datos que las ciencias aportan para el acceso y conocimiento por todos, con lo que se excluye lo que supone efectiva realidad inventiva que surge de la inspiración de los hombres. Por todo lo cual el concepto de plagio ha de referirse a las coincidencias estructurales básicas y fundamentales, y no a las accesorias, añadidas, superpuestas o modificaciones no transcendentales".

Por mucho que los demandados insistan en esto, es claro que, por la importancia contextual de los fragmentos copiados, sobre todo en cuento a información doctrinal, no actuaron conforme a los "usos honrados" ni los fines para haber actuado de esa manera estaban justificados, porque, en contra incluso de la esencia moral de alguno de los ejemplos expuestos en el libro, hubo una ocultación maliciosa de la fuente en los temas parcialmente plagiados, tomando y haciendo pasar por propio lo obtenido a través de un esfuerzo de persona ajena, en demérito de los legítimos intereses de este último.

La confrontación de lo reseñado, en la demanda, como copia de la tesis y ulterior publicación de la demandante con el libro del demandado revela que una parte de las coincidencias se refieren a citas y material doctrinal y bibliografía utilizada, lo que, indudablemente, en una tesis no es tarea propia o exclusiva del doctorando - caso de la actora- sino una labor en que de forma directa coopera el director, o, al menos tal es la función de la dirección de la tesis, esto es, facilitar o ilustrar al futuro doctor sobre aquellos textos, estudios y materiales que pudieran ser de interés a los fines de la investigación llevada a cabo. Es obvio, pues, que debemos descartar, por su propia esencia cualquier "plagio" vinculado a notas "al pie" y referencias y citas bibliográficas que obran en la tesis y en la ulterior publicación de la actora, pues en tal estado de cosas y dada la particular relación profesional (director de tesis-catedrático-/doctoranda) existente entre los hoy litigantes al tiempo de gestarse la obra de la que demandante deriva los derechos de propiedad intelectual por los que plantea la reclamación.

La obra de la actora es previa a la del demandado, y en la obra de éste, pese a ser director de la tesis de aquella no se cita la de la demandante ni siquiera en la bibliografía.

En definitiva, de todo ello, hay que extraer como conclusión que el demandado ha utilizado material de la actora, sin citar la fuente, ni siquiera en la bibliografía, utilizando conclusiones y estructura ofrecidas en la obra de aquella, sin que quede enervado, en cuanto a su eficacia, por el hecho de que el demandado dirigió aquella tesis, puesto que no ha probado, al no poder ser tenido en cuenta a tal fin el documento aportado, estudio alguno que anticipara la redacción, la estructura y las conclusiones recogidas por la demandante en su obra». 
condiciones que se hayan determinado entre las partes. Para ello se deberían considerar una serie de cláusulas, entre las cuales destacamos de mayor interés las siguientes.

En primer lugar habría que considerar los tipos de licencias empleadas, promocionando en particular las Creative Commons, que pueden resultar beneficiosas tanto para el docente como para el discente, pues al tiempo que se preservan los derechos de autor, se permite la copia, difusión e incluso la modificación de la obra, pero siempre reconociendo la autoría y bajo el amplio paraguas de condiciones posibles que Creative Commons permiten. Aún más, obteniendo una potencialmente mayor difusión de los trabajos.

Otro elemento de interés es facilitar la aceptación de la coautoría profesor(es) / alumno(s), facilitando el permiso para emplear el resultado en posteriores trabajos de índole académica, siempre citando la autoría.

Si es un trabajo desarrollado por el alumno en solitario, aparece una serie de elementos que deben valorarse. Por una parte, se eleva el riesgo de un posible plagio que, no siendo detectado por el director del trabajo, pueda estar presente en un porcentaje elevado del texto. Al margen del empleo de herramientas (Turnitin, Plagiarism) que permitan encontrar este tipo de situaciones, al no ser infalibles, una cláusula de autenticidad parece imponerse.

Crear una cláusula de confidencialidad si el resultado se va a aplicar en alguna empresa, siendo su difusión problemática en tanto puede revelar metodologías de trabajo, o se produce una circunstancia tal como la previsión de que vaya a formar parte de una patente en un futuro próximo o que los resultados del mismo se integran en un trabajo mayor, como un proyecto europeo, que no han llegado a su fase de difusión.

\section{Conclusiones}

Hemos visto que los conflictos se producen en el ámbito de la propiedad intelectual; sin embargo, no hay que olvidar que también surgen distintas cuestiones en torno a la protección en el ámbito de la propiedad industrial (Ramón, 2015: 50). 
Junto a ello, las denominadas malas prácticas no sólo se detienen en el plagio al que hemos hecho referencia, sino también al creciente incremento de la transacción económica de compraventa de trabajos, prácticas ambas totalmente denostadas en el ámbito académico (Comás et al., 2008: 5).

Hay que señalar, llegados a este punto, la abundancia de la oferta que, de modo más o menos clandestino en redes sociales y foros y, cada vez más, de forma abierta, aparece de forma que un alumno puede parecer que se "auto dirige", pero en realidad solo está pagando por un trabajo ajeno (colocando en una posición no muy agradable a su director académico en el caso de que él no perciba la realización del trabajo por terceros y otros, sí). Para muestra, tan solo con buscar los términos "compro Trabajo Final de Grado" en nuestro buscador favorito, encontraremos en las primeras posiciones páginas con nombres tan sugerentes como "elmejortfg", "tumatriculadehonor", "tutfg" o "apruebatodo.com".

Los sistemas de evaluación también influyen en el escenario que se plantea respecto a la necesidad de publicar, y ello influye ya no sólo en el alumnado, sino también en todo el profesorado (Ordorika, 2018: 463), es por ello que la propuesta de un documento que suscriban alumnado y profesorado en el ámbito del desarrollo de los trabajos académicos puede resolver, evitar y ser coherente con la protección de los derechos de autor de ambos, evitando que se produzcan situaciones descompensatorias y vulneración de los indicados derechos. También, de futuro, si el trabajo va a ser objeto de difusión científica, puede ser el documento un instrumento útil para determinar la participación de cada uno de los sujetos, así como, por ejemplo, el orden de firma y la delimitación de los derechos de cada uno.

\section{Referencias bibliográficas}

ALLEN, R. y SUMAN, G. (2018). «Liderazgo académico» y las condiciones del trabajo académico. Literatura: teoría, historia y crítica, 20(2), 293-319. Recuperado de http://www.scielo.org.co/scielo.php?script=sci_arttext\&pid=S0123$\underline{59312018000200293 \& \operatorname{lng}=\mathrm{es} \& \mathrm{nrm}=\mathrm{iso} \& \operatorname{tn} \mathrm{ln}=\mathrm{es}}$ 
BUENDÍA ESPINOSA, Mª. A., GARCÍA SALORD, S., GREDIAGA KURI, R., LANDESMANN, M., RODRÍGUEZ GÓMEZ, R., RONDERO LÓPEZ, N., RUEDA BELTRÁN, M. y VERA, H. (2017). Queríamos evaluar y terminamos contando: alternativas para la evaluación del trabajo académico. Perfiles educativos, 39(157), 200219.

Recuperado

de

http://perfileseducativos.unam.mx/iisue_pe/index.php/perfiles/article/view/58464/51367

CAMILLONI, A. (2019). La enseñanza del derecho orientada al desarrollo de la creatividad. Revista pedagogía universitaria y didáctica del Derecho, 6(1), 5-22. Recuperado de https://pedagogiaderecho.uchile.cl/index.php/RPUD/article/view/53743/56409

(Consultado el 13 de julio de 2019).

CANÓS DARÓS, L. y RAMÓN FERNÁNDEZ, F. (2007). La protección y regulación jurídica de los derechos de autor en la sociedad de la información. Revista General Informática de Derecho, 1-10.

CANÓS DAROS, L., RAMÓN FERNÁNDEZ, F. y ALBALADEJO FERNÁNDEZSILGADO, M. (2008). Los roles docentes y discentes ante las nuevas tecnologías y el proceso de convergencia europea. En: CD Resúmenes comunicaciones $V$ Congreso Iberoamericano de Docencia Universitaria (pp. 388-390). AIDU. Asociación Iberoamericana de Didáctica Universitaria. Valencia: Universidad Politécnica de Valencia e Instituto Ciencias de la Educación

CANÓS DARÓS, L. y RAMÓN FERNÁNDEZ, F. (2009). La calidad de la docencia respecto a las nuevas tecnologías y recursos didácticos. En: Autogestión, cooperación y participación en las Ciencias Sociales (pp. 341-352). Valencia: Universitat Politècnica de València.

CHAUHAN, V. and WILLETT, P. (2019). Academics' experience of copyright: A case study of teaching at the University of Greenwich. Global Knowledge, Memory and Communication, 68(6/7), 601-616. Recuperado de https://doi.org/10.1108/GKMC-01$\underline{2019-0010}$ 
CICERO, N. K. (2018). Innovar la enseñanza del derecho. ¿Solo se trata de Tecnologías de la Información y Comunicación?. Revista pedagogía universitaria y didáctica del

Derecho, $5(2)$, 91-109.

Recuperado de https://pedagogiaderecho.uchile.cl/index.php/RPUD/article/view/51976/54571

COMÁS FORGAS, R. y SUREDA NEGRE, J. (2008). El intercambio y compra-venta de trabajos académicos a través de internet. Edutec: Revista electrónica de tecnología educativa, 26, 1-16. Recuperado de https://www.edutec.es/revista/index.php/edutece/article/view/466/199

COMÁS FORGAS, R., SUREDA NEGRE, J. y OLIVER TROBAT, M. F. (2011). Prácticas de citación y plagio académico en la elaboración textual del alumnado universitario. Education in the knowledge society (EKS), 12(1), 359-385. Recuperado de http://revistas.usal.es/index.php/eks/article/view/7837/7863

DA SILVA, M. (2007). El trabajo académico del profesor del curso de medicina: contexto de cambio que expone contradicciones. REXE: Revista de estudios y experiencias en educación, 6(11), 99-112. Recuperado de https://dialnet.unirioja.es/ejemplar/179539

DÍAZ-VÁZZUEZ, R., GARCÍA-DÍAZ, A., MASIDE SANFIZ, J. M. y VÁZQUEZROZAS, E. (2018). El Trabajo Fin de Grado: fines, modalidades y estilos de tutorización. REDU: Revista de Docencia Universitaria, 16(2), Recuperado de https://polipapers.upv.es/index.php/REDU/article/view/10178

GÓMEZ LOZANO, Ma . del M. (2018). La propiedad intelectual sobre los trabajos académicos de los estudiantes universitarios. En: F. Carbajo Cascón y Ma . M. Curto Polo (Co.), Propiedad intelectual y transferencia de conocimiento en universidades y centros públicos de investigación (pp. 203-223). Valencia: Tirant lo Blanch.

LAHOZ GARCÍA, J. Ma . (2012). Consejos prácticos para la organización, elaboración y revisión de trabajos académicos. En: A. Fernández-Pampillón Cesteros, M. Goicoechea De Jorge, L. Hernández Yáñez y D. López García, D. (Co.), Filología y Tecnología: introducción a la escritura, la información, la información (pp. 19-28). Madrid: 
http://eprints.ucm.es/23457/1/Filologia2completoB.pdf

LICEA DE ARENAS, J., VICENTE RODRÍGUEZ, J., GÓMEZ, J.A. and ARENAS, M. (2004). Information literacy: implications for Mexican and Spanish university students. Library Review, 53(9), 451-460. Recuperado de https://doi.org/10.1108/00242530410565238

LÓPEZ PINO, L. (2008). El profesor universitario en la contemporaneidad. Revista Facultad de Ciencias Económicas: Investigación y Reflexión, 16(1), 23-40.

LÓPEZ PINO, L. (2010). Trabajo académico del profesor universitario: visión desde la investigación. Educación y Desarrollo Social, 4(2), 25-48. Recuperado de https://revistas.unimilitar.edu.co/index.php/reds/article/view/907/661

LOYARTE, E., GARCIA-OLAIZOLA, I., MARCOS, G., MORAL, M., GURRUTXAGA, N., FLOREZ-ESNAL, J. y AZUA, I., (2018). Model for calculating the intellectual capital of research centres. Journal of Intellectual Capital, 19(4), 787-813. Recuperado de https://www.emerald.com/insight/content/doi/10.1108/JIC-01-2017$\underline{0021 / \text { full/html }}$

LULL NOGUERA, C. y RAMÓN FERNÁNDEZ, F. (2017). Uso de herramientas digitales para mejorar la motivación en el aula universitaria. En: Innodoct/17. International Conference on Innovation, Documentation and Education (pp. 387-397). Valencia: Universitat Politècnica de València. Recuperado de https://riunet.upv.es/handle/10251/107064

LLOPIS NADAL, P. (2019). Estudiantes de ciencias jurídicas y trabajos de investigación: análisis de los derechos de propiedad intelectual sobre el TFG, el TFM y la Tesis Doctoral. Revista de Educación y Derecho - Education and law review, (20), 1-22. Recuperado de http://revistes.ub.edu/index.php/RED/article/view/29696/30024

MARÍN VICHIS, R. (2017). Errores frecuentes en el uso de la metodología de la investigación jurídica. Revista pedagogía universitaria y didáctica del Derecho, 4(2) 87- 
https://pedagogiaderecho.uchile.cl/index.php/RPUD/article/view/47972/50527

MELGUIZO MORENO, E. (2016). Los errores ortográficos en trabajos académicos de alumnos universitarios. En: A. E., Díez Mediavila, V. Brotons Rico, D. Escandelly J. Rovira Collado (Co.), Aprendizajes plurilingües y literarios: Nuevos enfoques didácticos (pp. 521-553). Alicante: Servicio de Publicaciones Universidad de Alicante Recuperado de http://rua.ua.es/dspace/handle/10045/64803

MIKELARENA PEÑA, F. (2007). La realización de trabajos académicos de investigación. Acciones e investigaciones sociales, 24, 211-259. Recuperado de https://papiro.unizar.es/ojs/index.php/ais/article/view/322

NARVÁEZ HERNÁNDEZ, J. R. (2019). Metodología crítica para la investigación científica del derecho. Revista pedagogía universitaria y didáctica del Derecho, 6(2), 8196.

https://pedagogiaderecho.uchile.cl/index.php/RPUD/article/view/55309/58861

NAVARRO FRÍAS, I. y MELERO BOSCH, L. (2018). Trabajos de investigación de contenido jurídico en titulaciones de perfil no jurídico dentro de las ciencias sociales. Revista de Educación y Derecho - Education and law review, 17, 1-31. Recuperado de http://revistes.ub.edu/index.php/RED/article/view/21875/23501

ORDORIKA SACRISTÁN, I. (2018). Las trampas de las publicaciones académicas. Revista española de pedagogía, 76(271), 463-480. Recuperado de https://revistadepedagogia.org/wp-content/uploads/2018/09/Las-trampas-de-laspublicaciones-acade\% $2 \% \mathrm{~A} 6 \% \mathrm{C} 3 \% \mathrm{BCmicas} . \mathrm{pdf}$

PACENZA, Ma . I. y ECHEVERRÍA, J. (2017). Sentidos del trabajo académico: Sobre la participación ad honorem de estudiantes en actividades académicas. Perspectivas en Psicología, $\quad$ 14(1), 40-50. Recuperado de http://www.seadpsi.com.ar/revistas/index.php/pep/article/view/325/pdf 
PADILLA GONZÁLEZ, L. E., JIMÉNEZ LOZA, L. y RAMÍREZ GORDILLO, Mª de los D. (2008). La satisfacción con el trabajo académico: motivaciones y condiciones del entorno institucional que la afectan. El caso de una universidad pública estatal. Revista mexicana de investigación educativa, 13(8), 843-856.__ Recuperado de http://perfileseducativos.unam.mx/iisue_pe/index.php/perfiles/article/view/40521/36883

PATHIRAGE, C., HAIGH, R., AMARATUNGA, D. and BALDRY, D. (2007). Enhancing the quality and consistency of undergraduate dissertation assessment: A case study. Quality Assurance in Education, 15(3), 271-286. Recuperado de https://doi.org/10.1108/09684880710773165

PÉREZ GARCÍA, Ma . A. (2008). Realización de trabajos académicamente dirigidos interdisciplinares en el marco del EEES. En: J. R. Flecha Andrés (Co.), El proceso de Bolonia y la enseñanza superior en Europa (pp. 157-166). Salamanca: Universidad Pontificia de Salamanca e Instituto de Estudios Europeos y Derechos Humanos.

PRÓSPER RIBES, J. y RAMÓN FERNÁNDEZ, F. (2019). MOOC y derechos de autor en la enseñanza online en el Derecho español. Revista La Propiedad Inmaterial, 27, 121147.

Recuperado de

https://revistas.uexternado.edu.co/index.php/propin/article/view/6075/7793

RAMÓN FERNÁNDEZ, F. (2013). La redefinición de las excepciones en materia de propiedad intelectual. Derecho de cita, plagio e internet. En: L. Cotino Hueso y L. Corredoira Alfonso (Co.), Libertad de expresión e información en internet. Amenazas y protección de los derechos personales (pp. 233-258). Madrid: Centro de Estudios Políticos y Constitucionales, Madrid.

RAMÓN FERNÁNDEZ, F. (2014). La realización de material audiovisual para los cursos masivos en abierto (MOOC): cuestiones legales no resueltas en el ámbito de la propiedad intelectual. IDP. Revista de Internet, Derecho y Política, 19, 78-91. Recuperado de https://idp.uoc.edu/articles/abstract/10.7238/idp.v0i19.2222/

RAMÓN FERNÁNDEZ, F. (2015). La ingeniería y la propiedad industrial en el ámbito universitario: marco legal y algunas dudas habituales. Revista La Propiedad Inmaterial, 
https://revistas.uexternado.edu.co/index.php/propin/article/view/4347/4931

RAMÓN FERNÁNDEZ, F. (2020). La producción científica en abierto y los repositorios institucionales como instrumentos de difusión y protección antiplagio. El caso de Riunet de la UPV. Revista PH. Instituto Andaluz de Patrimonio Histórico, 100, 152-154. Recuperado de http://www.iaph.es/revistaph/index.php/revistaph/article/view/4621

ROMERO NAVARRO, M. S. (2017). El trabajo académico en la construcción de saberes: entre el hacer y el discurso en la formación de profesores. Revista Iberoamericana para la Investigación y el Desarrollo Educativo. RIDE, 8(15), 735-752. Recuperado de https://www.ride.org.mx/index.php/RIDE/article/view/318/1514

SECUNDO, G., MARGHERITA, A., ELIA, G. y PASSIANTE, G. (2010). Intangible assets in higher education and research: mission, performance or both? Journal of Intellectual Capital, 11(2), 140-157. Recuperado de https://www.emerald.com/insight/content/doi/10.1108/14691931011039651/full/html

SCHOPFEL, J., VANACKER, S., KERGOSIEN, E. y JACQUEMIN, B. (2018) Master's theses and open scholarship: a case study.Digital Library Perspectives, 34(4), 276-287. Recuperado de https://www.emerald.com/insight/content/doi/10.1108/DLP-07-2018$\underline{0021 / f u l l / h t m l}$

TOLLER, F. M. (2010). Relevancia y sentido del buen trato a las fuentes del Derecho en la escritura jurídica. Díkaion: revista de actualidad jurídica, 19(2), 489-511. Recuperado de https://dikaion.unisabana.edu.co/index.php/dikaion/article/view/1790/2397

TOLLER, F. M. (2011). Propiedad intelectual y plagio en trabajos académicos y profesionales. Revista La Propiedad Inmaterial, 15, 85-97. Recuperado de https://revistas.uexternado.edu.co/index.php/propin/article/view/3000/2644

UBERTONE, F. P. (2017). Trabajos académicos: algunas cosas que no. Academia: revista sobre enseñanza del derecho de Buenos Aires, 29, 229-243. Recuperado de 
http://www.derecho.uba.ar/publicaciones/rev_academia/revistas/29/trabajos-academicosalgunas-cosas-que-no.pdf

URÍAS MARTÍNEZ, J. (1999). El estudio de derecho a través de la creación de materiales audiovisuales", Revista de Enseñanza Universitaria, 1, 441-450. Recuperado de http://institucional.us.es/revistas/universitaria/extra1999/art_38.pdf

VERA, H. (2017). La evaluación cuantitativa del trabajo académico: tres analogías", Sociológica (México), 32(90), 277-301. Recuperado de http://www.sociologicamexico.azc.uam.mx/index.php/Sociologica/article/view/1391/1179

VERDERA IZQUIERDO, B. (2014).“El aprendizaje y desarrollo del pensamiento jurídico a través de la jurisprudencia. Revista de Educación y Derecho - Education and law review, $10, \quad 1-22 . \quad$ Recuperado de http://revistes.ub.edu/index.php/RED/article/view/10711/13486 